\title{
Replicase-Mediated Resistance to Cucumber Mosaic Virus Does Not Inhibit Localization and/or Trafficking of the Viral Movement Protein
}

\author{
Tomas Canto and Peter Palukaitis \\ Virology Department, Scottish Crop Research Institute, Invergowrie, Dundee DD2 5DA, Scotland \\ Accepted 5 May 1999.
}

\begin{abstract}
Replicase-mediated resistance to cucumber mosaic virus (CMV) affects CMV replication and restricts $\mathrm{CMV}$ movement. Confocal laser scanning microscopic analysis of resistant plants inoculated with either CMV or potato virus $\mathrm{X}$, expressing the CMV movement protein (MP) fused to the green fluorescent protein (GFP), showed that the CMV MP was not inhibited from either plasmodesmal association or trafficking in the CMV-resistant plants. CMV expressing free GFP was able to move to adjacent cells, demonstrating that replicase-mediated resistance did not directly block the trafficking of CMV RNA.
\end{abstract}

Additional keywords: gating, gene silencing, pathogen-derived resistance, plasmodesmata.

Replicase-mediated resistance (RMR) is a form of pathogen-derived resistance in which either the polymerase gene or another viral gene associated with virus replication is expressed in a transgenic plant and elicits resistance to that virus and closely related strains (variants) of that virus (reviewed by Palukaitis and Zaitlin 1997). The replicase transgenes can encode either a functional or a dysfunctional protein. In most cases, the most resistant transgenic plants show low steadystate levels of transgene mRNA. However, resistant plants also show transcription rates comparable to those of nonresistant plants. These observations led to the concept of posttranscriptional gene silencing (PTGS) (reviewed by Baulcombe 1996a, 1996b). While the mechanisms of activation and maintenance of PTGS are not well understood, PTGS appears to apply to most cases of RMR, as well as pathogenderived resistance engendered by a number of other viralencoded genes (Baulcombe 1996a). In at least three cases of RMR, however, PTGS does not appear to be the primary cause of the resistance response. In the case of RMR to alfalfa mosaic virus, resistance appeared to be protein-mediated and occurred in plants with high steady-state levels of transgene mRNA (Brederode et al. 1995), while in the case of RMR engendered by either cucumber mosaic virus (CMV) RNA 1 (Canto and Palukaitis 1998) or a defective 2a polymerase gene encoded by CMV RNA 2 (Carr et al. 1994), virus replication

Corresponding author: Peter Palukaitis; Fax: 44-1382-562426; E-mail: ppaluk@scri.sari.ac.uk was severely reduced but not completely suppressed, as in PTGS. Moreover, in both forms of RMR involving CMV only limited cell-to-cell movement occurred, and long-distance (systemic) movement was blocked (Carr et al. 1994; Hellwald and Palukaitis 1995; Canto and Palukaitis 1998). In the case of RMR engendered by the defective CMV 2a polymerase gene, the block in long-distance movement was at the interface between bundle sheath cells and phloem-associated cells (Wintermantel et al. 1997). Both forms of RMR to CMV showed similar sequence specificities for resistance versus resistance breakage (Zaitlin et al. 1994; Canto and Palukaitis 1998), although the data did not support the view that a signal was transmitted long distance in the plant, inducing systemic acquired silencing (Wintermantel et al. 1997; Canto and Palukaitis 1998). In addition, the mapping of separate targets for inhibition of replication versus movement with CMV RNA 2 also indicated that RMR to CMV proceeded by mechanisms different from those engendered by PTGS (Hellwald and Palukaitis 1995).

CMV is a positive-sense RNA virus, the genome of which contains three RNAs encoding five genes (Palukaitis et al. 1992; Ding et al. 1994). The 1a and 2 a proteins, encoded by RNAs 1 and 2, respectively, are involved in CMV replication (Hayes and Buck 1990), while the $2 b$ protein, also encoded by RNA 2, appears to prevent host responses limiting virus accumulation and movement (Ding et al. 1995b; Brigneti et al. 1998). The $3 \mathrm{a}$ and $3 \mathrm{~b}$ proteins encoded by RNA 3 are the movement protein (MP) (Ding et al. 1995a; Kaplan et al. 1995) and the capsid protein (CP) (Habili and Francki 1974), respectively. Both the MP and $\mathrm{CP}$ are required for cell-to-cell movement in infected plants (Canto et al. 1997), while only the MP is needed to traffic viral RNA between cells in microinjection studies (Ding et al. 1995a).

The inhibition of CMV movement by resistant plants expressing the defective polymerase gene has also been analyzed by microinjection studies. The CMV MP was shown to be inhibited from trafficking itself or viral RNA, and from increasing the size exclusion limit for plasmodesmata (PD), which in nontransgenic plants allows gating of 9.4-kDa fluorescent dextran (Nguyen et al. 1996). These experiments suggested a direct effect of the defective replicase gene on viral movement either by blocking the normal pathway of delivery of MP to the PD or by hindering the interaction between MP 
and elements of the PD required for gating or trafficking. However, earlier data demonstrated that virus accumulated in numerous cells around the site of infection in the resistant plants (Carr et al. 1994; Hellwald and Palukaitis 1995; Wintermantel et al. 1997), indicating that limited cell-to-cell movement was occurring in both mesophyll and epidermal cells. On the other hand, in the microinjection studies, the tobacco mosaic virus (TMV) MP was also inhibited from trafficking viral RNA, but not from trafficking itself or from gating 9.4-kDa fluorescent dextran in the plants expressing RMR to CMV but not to TMV (Nguyen et al. 1996). Thus, to resolve these discrepancies, the effects of the defective 2a polymerase in RMR to CMV on MP localization and function were examined directly, after infection of the resistant plant with viruses expressing the jellyfish green fluorescent protein (GFP), either free or fused to the CMV MP.

The CMV MP, fused at its $\mathrm{C}$ terminus to the $\mathrm{N}$ terminus of the GFP and expressed from the potato virus X (PVX) vector (Fig. 1A), was shown previously to localize to PD, as did CMV MP itself (Blackman et al. 1998). When transgenic tobacco plants expressing the defective 2 a polymerase gene and showing RMR to CMV infection were inoculated with PVX RNA transcripts expressing the 3a-GFP fusion, PVX moved throughout the plants and showed localization of the 3a-GFP to $\mathrm{PD}$, as in nontransformed tobacco (Fig. 2B versus A). This observation indicates that in the CMV-resistant plants the MP was able to associate with whatever host components are required for directing or delivering the CMV MP to PD. However, these data do not demonstrate any functional association between the CMV MP and PD elements.

Previously, we showed that the 3a-GFP fusion could be expressed from CMV RNA 3 (Canto et al. 1997), after coinoculation of leaves with transcripts of CMV RNAs 1 plus 2 and the modified RNA 3 designated F:3a-GFP/CP RNA 3 (Fig. 1B). This virus was able to replicate in the inoculated cells of nontransformed tobacco and in most cases 3a-GFP was able to traffic to adjoining cells and localize to PD within the cell walls (Fig. 2C and Table 1), but the virus itself did not move cell to cell (Canto et al. 1997). Infection of transgenic tobacco expressing RMR to CMV by this same virus did not produce a green fluorescent signal (Fig. 2D), probably because the replication of the inoculated viral RNAs was inhibited (Hellwald and Palukaitis 1995). Thus, it was necessary to replace Fny-CMV RNA 2 with a chimeric RNA 2 able to replicate to normal levels in protoplasts of the resistant plants, but not able to overcome the resistance against virus move-

\section{A PVX:3a-GFP RNA}

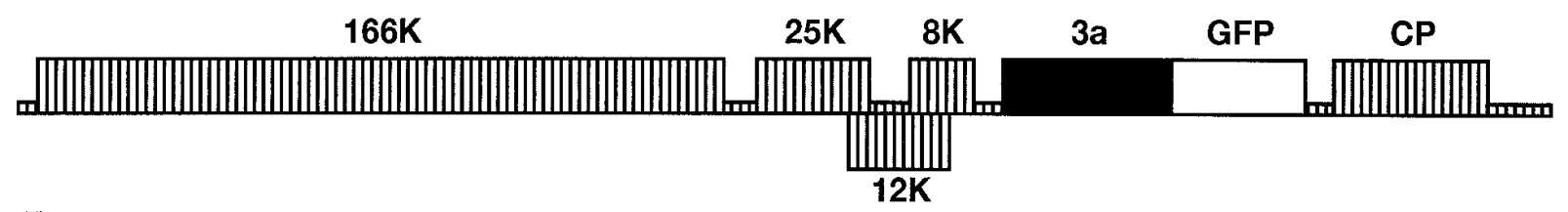

B pF:3a-GFP/CP

RNA3

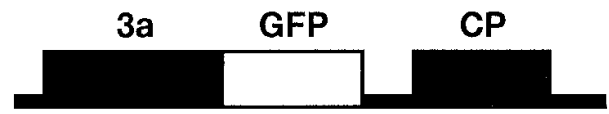

C pK-NHd

RNA2
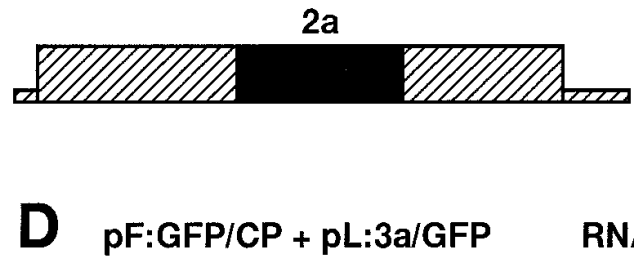

RNA3

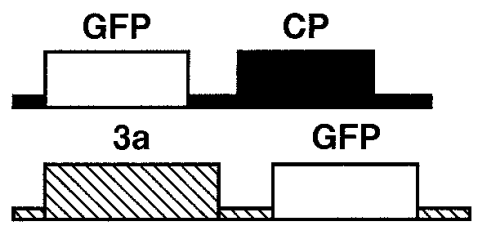

Fig. 1. Schematic representation of cDNA constructs. A, Potato virus X (PVX) RNA, modified to express the 3a movement protein of cucumber mosaic virus (CMV) fused at its $\mathrm{C}$ terminus to the green fluorescence protein (GFP). The various PVX genome genes are indicated; $\mathrm{CP}=$ capsid protein. $\mathbf{B}$, FnyCMV RNA 3, modified to express the 3 a movement protein fused at its $\mathrm{C}$ terminus to the GFP. C, A CMV RNA chimera derived from RNA 2 of the Fnyand the K- strains of CMV (solid and hatched boxes, respectively; Hellwald and Palukaitis 1995). D, Fny-CMV RNA 3 with the GFP replacing the 3a gene, plus LS-CMV RNA 3 with the GFP replacing the CP gene. This combination of RNAs 3 shows complementation of gene functions leading to cellto-cell movement (Canto et al. 1997). 


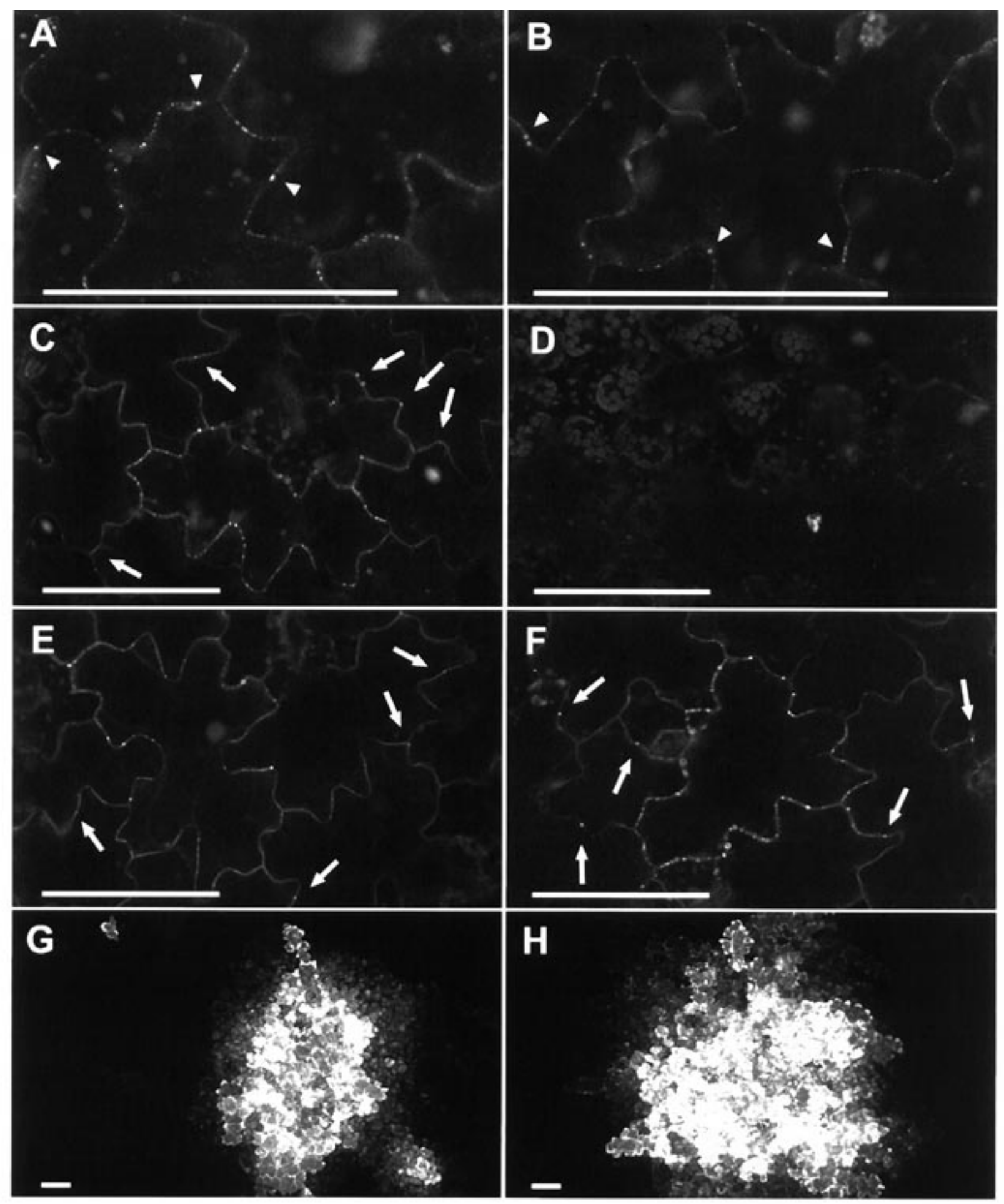

Fig. 2. Distribution of green fluorescence protein (GFP)-derived fluorescence in tobacco epidermis inoculated with viral RNAs expressing the $g f p$ gene. $\mathbf{A}$ and $\mathbf{B}$, Potato virus X (PVX) expressing the 3 a movement protein of cucumber mosaic virus (CMV) fused at its $\mathbf{C}$ terminus to the GFP. C and D, FnyCMV transcript RNAs 1 and 2, plus RNA 3 derived from construct pF:3a-GFP/CP, that expresses the 3a protein fused at its $\mathrm{C}$ terminus to the GFP. E and F, Fny-CMV transcript RNA 1, plus RNA 2 derived from construct pK-NHd, that expresses a chimera between the Fny- and K- strains of CMV, and RNA 3 derived from construct pF:3a-GFP/CP that expresses the 3a protein fused at its C terminus to the GFP. $\mathbf{G}$ and $\mathbf{H}$, Fny-CMV transcript RNA 1, plus RNA 2 derived from construct pK-NHd, plus a mixture of RNAs 3 derived from constructs pF:GFP/CP and pL:3a/GFP, in which the GFP gene replaces the 3a or the capsid protein genes, respectively. A, C, E, and $\mathbf{G}$ correspond to nontransformed tobacco. B, D, F, and $\mathbf{H}$ correspond to the R2-2 transgenic line of CMV-resistant tobacco. A-C, E, and $\mathbf{F}$ show the presence of fluorescence derived from the 3a-GFP fusion protein localized in discrete spots in the walls between infected cells (arrowheads in $\mathbf{A}$ and $\mathbf{B}$ ), as well as in the walls of some of the adjacent cells $(\operatorname{arrows}$ in $\mathbf{C}, \mathbf{E}$, and $\mathbf{F}$ ). $\mathbf{G}$ and $\mathbf{H}$ show the presence of fluorescence derived from the expression of free GFP in clusters composed of hundreds of epidermal and mesophyl cells. Images were obtained with a confocal laser scanning microscope. The bars represent $100 \mu \mathrm{m}$. 
ment. This chimeric RNA 2, K-NHd RNA 2 (Fig. 1C), contains the central third of Fny-CMV RNA 2 and the flanking sequences derived from the resistance-breaking K-CMV RNA 2 (Hellwald and Palukaitis 1995). In combination with FnyCMV RNA 1 and F:3a-GFP/CP RNA 3 (Fig. 1B), K-NHd RNA 2 was able to accumulate in the inoculated epidermal cells of the CMV-resistant transgenic plants, and green fluorescence could be detected in the PD of such infected cells. In the majority of cases, green fluorescence was also seen in the PD of the adjacent cells, distal to the infected cell (Fig. 2F and Table 1). This indicates that in the resistant plants the 3aGFP was able to associate with host factors in the PD of the inoculated cells, traffic MP-GFP through those cells to adjacent cells, and become associated with the PD of the adjacent cells, just as in nonresistant plants (Fig. 2E). Thus, although the defective polymerase or its mRNA did not inhibit the trafficking of the 3a-GFP from cell to cell, these data do not address the issue of whether the defective replicase or its mRNA could prevent the cell-to-cell trafficking of viral RNA by the CMV MP.

To ascertain whether the defective replicase gene product inhibited the movement of viral RNA, a different inoculum was prepared. This consisted of a mixture of four CMV RNAs, able to express free GFP and move cell to cell during infection of nontransformed plants (Fig. 2G). This mixture of RNAs consists of Fny-CMV RNA 1, K-NHd RNA 2, and the two hybrid RNA 3s, F:GFP/CP RNA 3 and L:3a/GFP RNA 3 (Fig. 1D). A similar combination of RNAs (containing FnyCMV RNA 2 instead of K-NHd RNA 2) was shown previously to move cell to cell in tobacco, but not to move systemically (Canto et al. 1997). Infection of the resistant plants expressing the defective $2 \mathrm{a}$ polymerase gene by these RNAs resulted in similar accumulation (of GFP) and movement profiles as in cells of nontransformed plants (Fig. $2 \mathrm{H}$ versus $\mathrm{G}$ ). Thus, the defective 2 a polymerase did not directly prevent the cell-to-cell movement of the CMV RNAs to cells adjacent to the infection site.

Why do our results with infection differ from those of Nguyen et al. (1996) with microinjection? It is unlikely to be due to differences between results obtained in epidermal cells (this report) versus mesophyll cells (Nguyen et al. 1996), since in situ hybridization analysis of chlorotic lesions from plants infected by Fny-CMV showed that virus accumulation

Table 1. Effect of replicase-mediated resistance to cucumber mosaic virus (CMV) on the movement of 3a-GFP to adjacent cells

\begin{tabular}{lcc}
\hline $\begin{array}{l}\text { CMV transcript } \\
\text { RNA source }\end{array}$ & $\begin{array}{c}\text { Nontransformed } \\
\text { tobacco }\end{array}$ & $\begin{array}{c}\text { CMV-resistant } \\
\text { tobacco }^{\mathbf{a}}\end{array}$ \\
\hline F1 F2 pF:3a-GFP/CP & $22 / 25^{\mathrm{c}}$ & Not detected \\
F1 pK-NHd pF:3a-GFP/CP & $20 / 25$ & $19 / 25$ \\
\hline
\end{tabular}

a Transgenic tobacco line R2-2 (Carr et al. 1994) expressing a defective polymerase gene of Fny-CMV RNA 2.

${ }^{\mathrm{b}}$ F1 and F2 represent Fny-CMV transcript RNAs 1 and 2. pK-NHd represents a chimeric RNA 2 transcript generated from RNA 2 of the Fnyand K- strains of CMV (Hellwald and Palukaitis 1995). F:3a-GFP/CP represents an Fny-CMV transcript RNA 3 that expresses the 3a protein fused at its $\mathrm{C}$ terminus to the green fluorescence protein (GFP; Canto et al. 1997).

${ }^{c}$ Number of infection foci where GFP-derived fluorescence was detected in adjacent cells, over the total number of GFP-fluorescent infection foci. Cells were screened by confocal laser scanning microscopy at 3 days post inoculation. (and hence replication and movement) occurred in both epidermal and mesophyll cells (Wintermantel et al. 1997). Moreover, virus movement into mesophyll cells was also observed here (Fig. 2H). Thus, while there may be a barrier to movement from bundle sheath to phloem-associated cells (Wintermantel et al. 1997), this barrier does not exist in either mesophyll or epidermal cells. The limitation of virus spread to the chlorotic lesion could be due to either an elicited host response or a gradual effect that is not detectable within the first few cells infected.

Microinjection studies involving the TMV MP and the CMV-resistant transgenic plants also showed an inhibition of RNA trafficking, but not trafficking of TMV MP per se or gating of $9.4-\mathrm{kDa}$ fluorescent dextran by the TMV MP (Nguyen et al. 1996). To explain this unexpected observation (since the CMV-resistant plants are not resistant to TMV), Nguyen et al. (1996) suggested that some component associated with TMV replication must be required for TMV movement in such plants. By the same reasoning, it could also be argued that some component of CMV is necessary for trafficking of RNA by the CMV MP. This CMV component would not be necessary for the MP to associate with PD, since the CMV MP-GFP expressed from PVX was still able to localize to PD. However, the CMV component could facilitate cell-to-cell movement of the MP:RNA complexes in infected plants. Such a role could be played by the viral CP, which is needed for cell-to-cell movement in plants (Canto et al. 1997), but not in microinjection studies (Ding et al. 1995a). This is yet another example of conclusions derived from microinjection experiments that clearly contradict those obtained from infectivity experiments. Alternatively, such a function may be provided by either the 2 a protein or RNA 2 itself, but it is unlikely to be provided by the $2 \mathrm{~b}$ protein, since CMV containing RNA 2 of K-NHd would express the K-CMV RNA 2 2b gene, and is still restricted in movement (Hellwald and Palukaitis 1995). Thus, these observations show that care must be taken in interpreting conclusions derived solely from microinjection studies, and such studies should always be supported by biological data from infectivity experiments, to allow for effects by other viral gene products.

\section{ACKNOWLEDGMENTS}

We thank Petra Boevink and Simon Santa Cruz for supplying the PVX vector expressing 3a-GFP, and Simon Santa Cruz for his helpful comments on this manuscript. This work was supported by grant-inaid from Scottish Office Agriculture, Environment and Fisheries Department.

\section{LITERATURE CITED}

Baulcombe, D. C. 1996a. Mechanism of pathogen-derived resistance to viruses in transgenic plants. Plant Cell 8:1833-1844.

Baulcombe, D. C. 1996b. RNA as a target and an initiator of posttranscriptional gene silencing in transgenic plants. Plant Mol. Biol. 32: 79-88.

Blackman, L. M., Boevink, P., Santa Cruz, S., Palukaitis, P., and Oparka, K. J. 1998. The movement protein of cucumber mosaic virus traffics into sieve elements in minor veins of Nicotiana clevelandii. Plant Cell 10:525-537.

Brederode, F. T., Taschner, P. E. M., Posthumus, E., and Bol, J. 1995. Replicase-mediated resistance to alfalfa mosaic virus. Virology 207: 467-474.

Brigneti, G., Voinnet, O., Li, W.-X., Ji, L.-H., Ding, S.-W., and Baul- 
combe, D. C. 1998. Viral pathogenicity determinants are suppressors of transgene silencing in Nicotiana benthamiana. EMBO J. 17:67396746.

Canto, T., and Palukaitis, P. 1998. Transgenically expressed cucumber mosaic virus RNA 1 simultaneously complements replication of cucumber mosaic virus RNAs 2 and 3 and confers resistance to systemic infection. Virology 250:325-336.

Canto, T., Prior, D. A. M., Hellwald, K.-H., Oparka, K. J., and Palukaitis, P. 1997. Characterization of cucumber mosaic virus. IV Movement protein and coat protein are both essential for cell-to-cell movement of CMV. Virology 237:237-248.

Carr, J. P., Gal-On, A., Palukaitis, P., and Zaitlin, M. 1994. Replicasemediated resistance to cucumber mosaic virus in transgenic plants involves suppression of both virus replication in the inoculated leaves and long-distance movement. Virology 199:439-447.

Ding, B., Li, Q., Nguyen, L., Palukaitis, P., and Lucas, W. J. 1995a. Cucumber mosaic virus 3 a protein potentiates cell-to-cell trafficking of CMV RNA in tobacco plants. Virology 207:345-353.

Ding, S.-W., Anderson, B., Haase, H., and Symons, R. H. 1994. New overlapping gene encoded by the cucumber mosaic virus genome. Virology 198:593-601.

Ding, S.-W., Li, W.-X., and Symons, R. H. 1995b. A novel naturally occurring hybrid gene encoded by a plant RNA virus facilitates long distance virus movement. EMBO J. 14:5762-5772.

Habili, N., and Francki, R. I. B. 1974. Comparative studies on tomato aspermy and cucumber mosaic viruses. III. Further studies on rela- tionship and construction of a virus from parts of the two viral genomes. Virology 61:443-449.

Hayes, R. J., and Buck, K. W. 1990. Complete replication of a eukaryotic virus RNA in vitro by a purified RNA-dependent RNA polymerase. Cell 63:363-368.

Hellwald, K.-H., and Palukaitis, P. 1995. Viral RNA as a potential target for two independent mechanisms of replicase-mediated resistance against cucumber mosaic virus. Cell 83:937-946.

Kaplan, I. B., Shintaku, M. H., Li, Q., Zhang, L., Marsh, L. E., and Palukaitis, P. 1995. Complementation of virus movement in transgenic tobacco expressing the cucumber mosaic virus $3 \mathrm{a}$ gene. Virology 209:188-199.

Nguyen, L., Lucas, W. J., Ding, B., and Zaitlin, M. 1996. Viral RNA trafficking is inhibited in replicase-mediated resistant transgenic tobacco plants. Proc. Natl. Acad. Sci. USA 93:12643-12647.

Palukaitis, P., Roossinck, M. J., Dietzgen, R. G., and Francki, R. I. B. 1992. Cucumber mosaic virus. Adv. Virus Res. 41:281-348.

Palukaitis, P., and Zaitlin, M. 1997. Replicase-mediated resistance to plant virus disease. Adv. Virus Res. 48:349-377.

Wintermantel, W. M., Banerjee, N., Oliver, J. C., Paolillo, D. J., and Zaitlin, M. 1997. Cucumber mosaic virus is restricted from entering minor veins in transgenic tobacco exhibiting replicase-mediated resistance. Virology 231:248-257.

Zaitlin, M., Anderson, J. M., Perry, K. L., Zhang, L., and Palukaitis, P. 1994. Specificity of replicase-mediated resistance to cucumber mosaic virus. Virology 201:200-205. 